\title{
Attitude and Awareness about Radiation Protection among Dental Surgeons in Jaipur City: A Questionnaire Based Study
}

\section{Kanica Sharma ${ }^{1 *}$, Neelkant Patil ${ }^{2}$, Shobhit Kaswan ${ }^{3}$, Lalita Chandna ${ }^{3}$, Sunbul Tabrez ${ }^{4}$ and Dinesh Kumar ${ }^{4}$}

\author{
${ }^{1}$ Post-Graduate Student, Oral Medicine and Radiology at Rajasthan Dental College \\ and Hospital, Jaipur, Rajasthan, India \\ ${ }^{2}$ Head of the Department, Oral Medicine and Radiology, Rajasthan Dental College \\ and Hospital, Jaipur, Rajasthan, India \\ ${ }^{3}$ Senior Lecturer, Oral Medicine and Radiology, Rajasthan Dental College and \\ Hospital, Jaipur, Rajasthan, India \\ ${ }^{4}$ Post-Graduate Student, Rajasthan Dental College and Hospital, Jaipur, Rajasthan, \\ India \\ *Corresponding Author: Kanica Sharma, Post-Graduate Student, Oral Medicine and \\ Radiology at Rajasthan Dental College and Hospital, Jaipur, Rajasthan, India.
}

Received: February 18, 2021

Published: March 15, 2021

(C) All rights are reserved by Kanica Sharma., et al.

\section{Abstract}

Introduction: Dental radiology represents the most frequent diagnostic radiological investigation performed in almost every age groups.

Aim and Objective: The aim of this study was to determine the level of awareness regarding radiation protection among the dental practitioners in Jaipur city.

Materials and Methods: This questionnaire study was carried out among 100 dental surgeons in Jaipur city. A multiple-choice response type of questionnaire was designed with close - ended questions with a maximum choice of 3 responses.

This questionnaire study highlights important parameters that must be taken into consideration to reduce radiation exposure and also show the level of awareness and concern of dental practitioners of Jaipur regarding hazards and protection.

Results: Out of 100 dentists who participated in the study, 72\% considered clinical examination necessary before prescribing the dental radiograph, only $4 \%$ dentists adviced radiographs to pregnant dental patients. Approximately $90 \%$ of the dental practitioners were aware of the ALARA principle, about $80 \%$ of the dentists used E-speed film in their clinic, nearly $53 \%$ did not get their X-ray machine AERB approved and only a small amount i.e. 08\% use dosimeter to measure the radiation dose.

Conclusion: The results indicate that a majority of the dental practitioners are not aware of the safety protection measures. They should stay informed of new information on radiation safety issues as well as developments in equipment and techniques to improve radiographic practice.

Keywords: Awareness; Dental Surgeons; Radiation Protection; Dental Radiograph 


\section{Introduction}

Radiographic examination is an important part of dental practice as it serves as a prime source of investigation and helps us to arrive at a proper diagnosis [1].

Radiation has two types of effects on living systems i.e. direct and indirect effects. In direct effects, the energy of a photon or secondary electron ionizes biologic macromolecules, whereas in indirect effect there is an interaction with water and organic molecules and production of unstable free radicals [2].

These free radicals play an important role in producing molecular changes in the DNA strands and further cause damage to the living tissues. To prevent and reduce such effects, it is necessary to have a thorough knowledge about the exposure parameters and radiation protection measures.

Although the amount of radiation exposure experienced in dentistry is minimal, it emposes stochastic effects. There is a definite need to identify the barriers which prevent the application of radiation protection guidelines among dental professionals [3].

\section{Aim of the Study}

The aim of this study was to determine the level of awareness regarding radiation protection among the dental practitioners in Jaipur city.

\section{Materials and Methods}

A questionnaire survey was carried out among 100 dental practitioners in Jaipur with close-ended questions and a maximum choice of 3 responses comprising of Demographic characteristics, Radiographic equipment and techniques and Methods used for patient and personnel protection

\section{Procedure}

A printed copy of this structured questionnaire of 20 questions was produced before dental practitioners of Rajasthan dental college and hospital for recording their responses (Figure 1). Electronic format of this questionnaire was also prepared to collect responses from local dental practitioners of Jaipur city having their own Dental clinic. Seventy responses were collected in person from the dentists and 30 were received in the electronic format. Attempting each question was mandatory for each responder. Only one option was to be marked. The data had been collected on the terms to maintain the anonymity of the practitioners. The questionnaires were collected, compiled and evaluation of their responses was done. Data collected were then stored in the e-format in excel sheet.

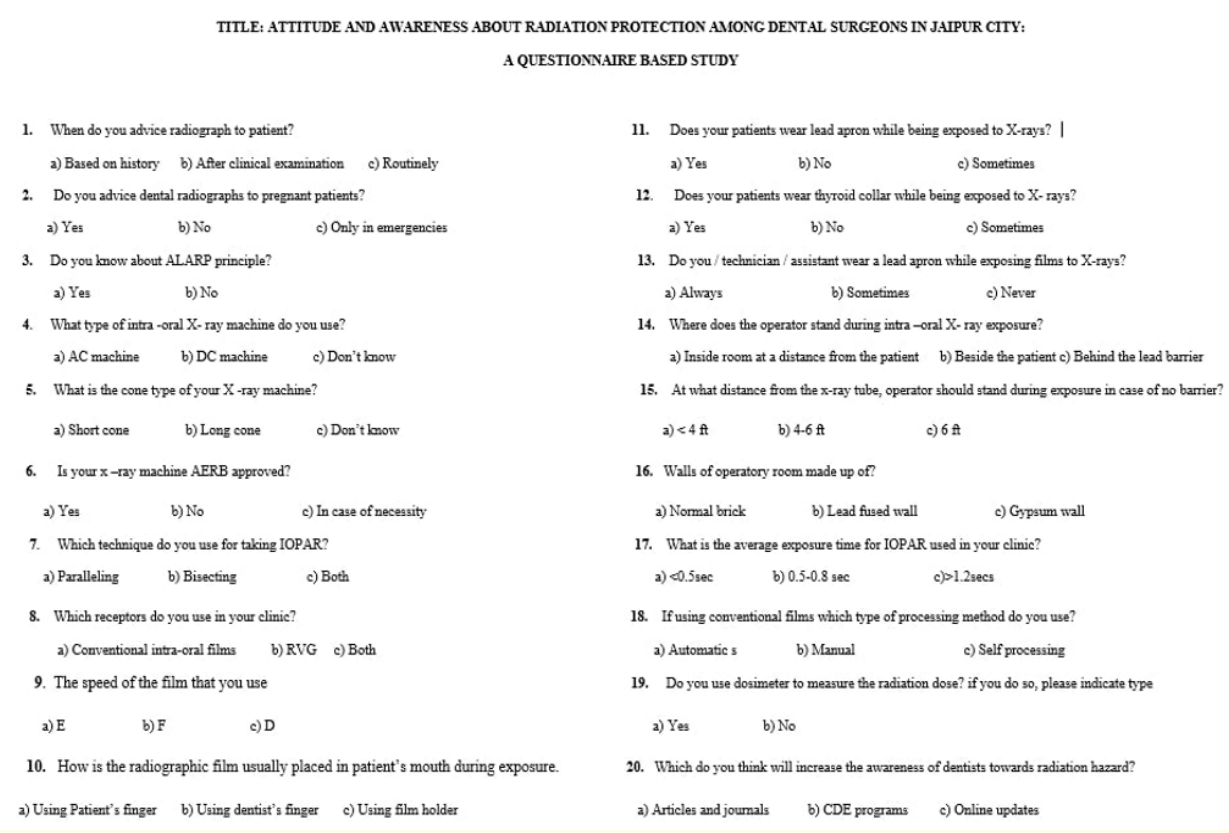

Figure 1: Structured questionnaire of multiple-choice questions. 


\section{Results}

Out of 100 dentists who participated in the study, $72 \%$ considered clinical examination necessary before prescribing the dental radiograph, while $24 \%$ prescribe radiographs based on patient's history and only $4 \%$ prescribed radiograph on a routine basis (Figure 2). Only $4 \%$ dentists adviced radiographs to pregnant dental patients and 55\% did not advice while $41 \%$ prescribe radiographs only in emergencies. approximately $90 \%$ of the dental practitioners were aware of the ALARA principle while $10 \%$ were unaware of this principle. A fraction of $40 \%$ of the practitioners have their X-ray machine AERB approved while 53\% did not get their x-ray machine AERB approved and only 7\% did it only in case of necessity (Figure 3). About $80 \%$ of the dentists used E-speed film in their clinic while only $2 \%$ used F speed film and $18 \%$ used D speed films. About $17 \%$ of dentists use conventional films, $59 \%$ use radio-visio graphy whereas $24 \%$ are using both types of receptors. $20 \%$ of the dentists use film holders to place the radiographic film in the patient mouth during exposure, $67 \%$ uses patient finger while about $13 \%$ uses their finger to hold the radiographic film. Almost $72 \%$ of the dental practitioners follow the distance -object rule i.e. they stand at a distance of approx 6 feet away during exposure. 92\% of the dental practitioners do not use dosimeter to measure the radiation dose while $08 \%$ use to measure radiation dose.

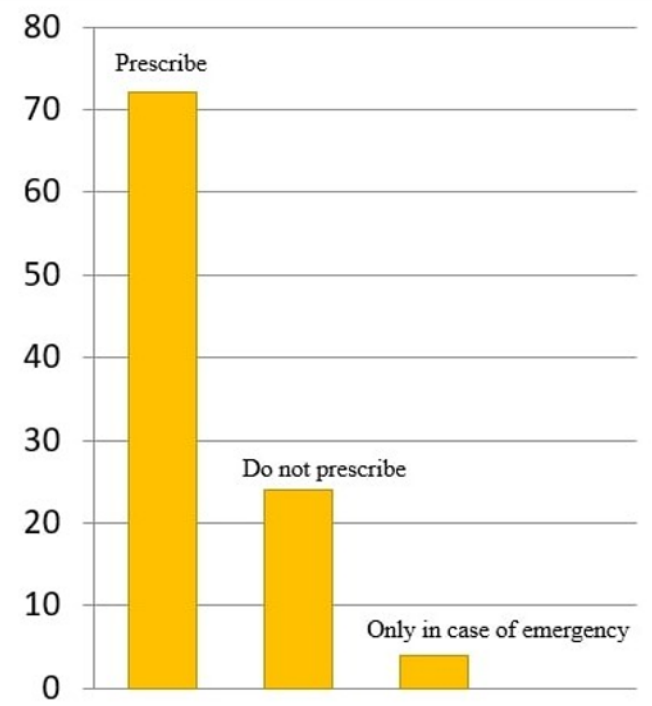

Figure 2: Radiographs prescribed in dentistry.

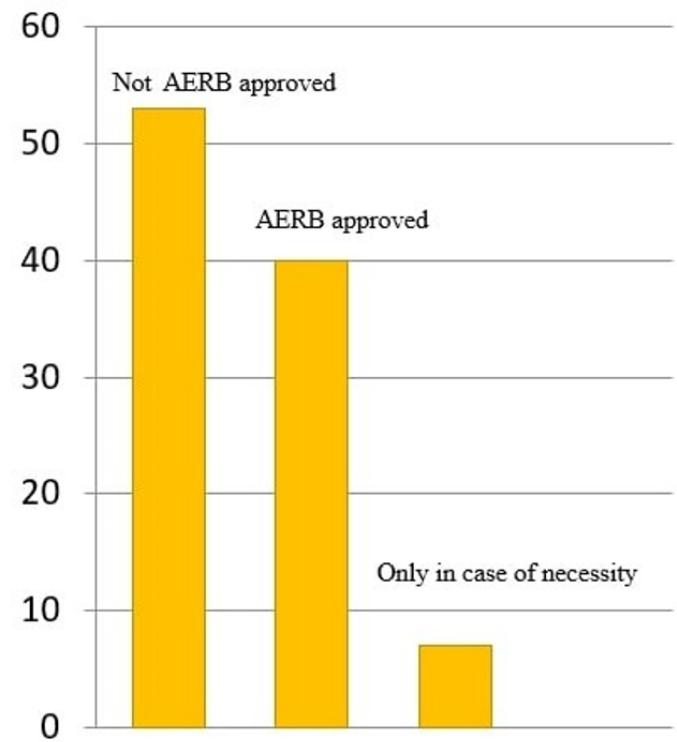

Figure 3: AERB approved x-ray machine.

\section{Discussion}

Although the amount of radiation exposure experienced in dentistry is minimal, its adverse effects cannot be excluded. There is a definite need to identify the barriers which prevent the application of radiation protection guidelines among dental professionals [3].

In our present study, $72 \%$ considered clinical examination and history taking necessary before prescribing the dental radiograph which was quite similar to the study conducted by Javali., et al. (2018) which resulted as $98.5 \%$ [4].

Radiation exposure to pregnant women results in various biological effects on foetus including developmental abnormalities, mutagenic carcinogenic effects and intrauterine death [5].

In pregnancy, it is advised to avoid exposure to radiation in the first trimester ( $8-15^{\text {th }}$ week of pregnancy), it is better to perform radiological investigation in the second and third trimester under proper protection i.e. use of lead apron, thyroid collar, etc [6].

Our study reveals that only $4 \%$ dentists advice radiographs to pregnant dental patients and $41 \%$ prescribe radiographs only in emergencies. 
Good radiographic procedure includes the use of the fastest image, the use of these films aids in decreasing the patient exposure. E- and F-speed films are double times faster than the D speed films. Our study showed that most of the practitioners who were using conventional radiographic films, (80\%) were using E-speed films, $18 \%$ used D-speed films and only $2 \%$ used F- films which was in agreement with the previous studies done by Swarna., et al, Kaviani., et al. and Chaudhry., et al. who have reported the usage of Espeed films in private dental practice to be up to 74,70 , and $69 \%$, respectively [7-9].

The safe distance of $6 \mathrm{ft}$ and more was adhered to by most of the dental practitioners in our study (72\%). We also found a shocking data that $50 \%$ of the dentists were standing beside the patients while making radiographs, which was very high as compared with the study done by Kumar., et al. (2018) involving only $10.5 \%$. In our study, we found that very few dentists $(27 \%)$ used lead aprons for their patients.

Biological effects of ionizing radiation are grouped into stochastic and deterministic effects. In deterministic effect, radiation above a particular determined dose starts causing biological damage whereas on the other hand in the case of stochastic effect there is no particular dose level, also called as all -or -none -phenomenon. Dentists as well as patients are more prone to the risk of stochastic effect because of lack of dose threshold limit [10].

In present study only (08\%) of dental professionals used personal monitoring which is the best way to ensure that personnel are following recommended safety protection measures. Several types of dosimeters including the thermo luminescence dosimeter, photoluminescence glass dosimeter, or optical stimulated luminescence dosimeter could be used to measure the exposure [11]. One the limitations of a study of this nature is the possibility of bias. It is possible that some answers may represent the ideal situations intended by the respondents, but adherence to these standards may be negligible at times or in certain areas of practice. Thus, variation in standards may occur within dental clinics, which do not match with our results.

\section{Conclusion}

Considering the results of this questionnaire study, it can be concluded that only a small fraction of dental practitioners is using dosimeters, have their x-ray machine AERB approved and very few of them are aware about the most recent trends in radiation protection.

\section{Key Message}

It's the need of the hour for dental practitioners to keep themselves updated regarding the potential of radiation hazards and safety protocols by attending more workshops and short-term training courses to protect themselves, their staff as well as the patients.

\section{Bibliography}

1. White SC., et al. "Parameters of radiologic care: An official report of the American Academy of Oral and Maxillofacial Radiology". Oral Surgery, Oral Medicine, Oral Pathology, Oral Radiology, and Endodontology 91 (2001): 498-511.

2. White SG and Pharoah MJ. "Oral radiology: principles and interpretation". (5 $5^{\text {th }}$ edition) Mosby, St. Louis, USA (2004): 25-46.

3. Reddy RK., et al. "Evaluation of knowledge and awareness on practice of dental radiographic safety measures in West Godavari District, India - A Questionnaire Based Cross- Sectional Study 1.2 (2017): 03-09.

4. Javali R and Dantu R. "Attitude and awareness about radiation protection among dental surgeons in North Karnataka: A Questionnaire Study". 30.2 (2018).

5. Bent RL. "The effect of embryonic and fetal exposure to X-ray, microwaves, and ultrasound. Counseling the pregnant and nonpregnant patient about these risks". Seminars in Oncology 16 (1989): 347-348.

6. White SC and Pharoah MJ. "Oral Radiology: Principles and Interpretation". $6^{\text {th }}$ edition. St. Louis, USA: Mosby (2009).

7. Math SY., et al. "Compliance of Indian dentists with oral radiology safety measures". Journal in Oral Radiology and Maxillofacial Radiology 1 (2013): 104-110.

8. Eskandarlou A., et al. "Observance of radiation protection principles in Iranian dental schools". Journal of Research in Medical Sciences 15 (2010): 292-293.

9. Kaviani F., et al. "Evaluation of X-ray protection methods used in dental offices in Tabriz in 2005-2006". Journal of Dental Research, Dental Clinics, Dental Prospects 1 (2007): 49-52. 
10. International Commission on Radiological Protection. The 2007 recommendation of the International Commission on Radiological Protection, ICRP publication 103". Ann ICRP 1.3 (1977): 1-332.

11. Math Swarna Yerebairapura., et al. "Compliance of Indian Dentists with Oral Radiology Safety Measures". Journal of Oral and Maxillofacial Radiology 1.3 (2013): 104-110.

\section{Assets from publication with us}

- Prompt Acknowledgement after receiving the article

- Thorough Double blinded peer review

- Rapid Publication

- Issue of Publication Certificate

- High visibility of your Published work

Website: www.actascientific.com/

Submit Article: www.actascientific.com/submission.php

Email us: editor@actascientific.com

Contact us: +919182824667 